\title{
High MICB expression as a biomarker for good prognosis of colorectal cancer
}

\author{
Qingyang Feng ${ }^{1}$. Shanchao $\mathrm{Yu}^{1} \cdot$ Yihao Mao ${ }^{1}$ Meiling $\mathrm{Ji}^{1} \cdot$ Ye Wei ${ }^{1} \cdot$ Guodong He ${ }^{1}$. Wenju Chang ${ }^{1}$. Dexiang Zhu ${ }^{1}$. \\ Li Ren ${ }^{1}$. Jianmin $\mathrm{Xu}^{1}{ }^{1}$
}

Received: 25 December 2019 / Accepted: 17 February 2020 / Published online: 18 April 2020

(c) The Author(s) 2020

\begin{abstract}
Introduction Major histocompatibility complex (MHC) plays an important role in colorectal cancer (CRC) immunity. However, the function of MHC class I chain-related B (MICB) molecule is not very clear. In this study, we explored the prognostic effect of MICB in colorectal cancer.

Material and methods From 2008-05 to 2012-11, consecutive CRC patients of Zhongshan Hospital, Fudan University were retrospectively enrolled as primary cohort. The inclusion criteria were as follows: receiving primary radical resection, pathologically confirmed colorectal adenocarcinoma, no treatment before surgery, clinicopathological data available. Another cohort of CRC patients were collected from a public dataset GSE39582 of GEO database from 1987 to 2007 in the same criteria for validation. MICB was detected using immunochemistry and evaluated as prognostic biomarker. The cut-off value of MICB expression was calculated using X-tile software.

Results Finally, 863 patients were enrolled in the primary cohort, and 556 patients were enrolled in the validation cohort. MICB expression was significantly associated with tumor size and primary histological type in primary cohort, and with primary tumor location and distant metastases in validation cohort. The survival analysis showed that patients with high MICB expression had significantly better overall survival in both primary $(P=0.002)$ and validation $(P=0.001)$ cohorts. The multivariate analysis also confirmed that high MICB expression was a significantly independent protective factor for overall survival in both primary (hazard ratio $\mathrm{HR}=0.741,95 \% \mathrm{CI} 0.594-0.924$ ) and validation ( $\mathrm{HR}=0.699,95 \%$ CI $0.508-0.961$ ) cohorts.

Conclusion For stage I-IV CRC patients, MICB was confirmed a novel independent prognostic factor. It could help better stratification of CRC prognosis.
\end{abstract}

Keywords MICB $\cdot$ Colorectal cancer $\cdot$ Prognosis

\section{Abbreviations}

CRC Colorectal cancer

MHC Major histocompatibility complex

Qingyang Feng, Shanchao Yu, Yihao Mao and Meiling Ji contributed equally to this article.

Electronic supplementary material The online version of this article (https://doi.org/10.1007/s00432-020-03159-0) contains supplementary material, which is available to authorized users.

$\checkmark$ Li Ren

renli1969@foxmail.com

Jianmin Xu

xujmin@aliyun.com

1 Department of General Surgery, Zhongshan Hospital, Fudan University, Shanghai, China
MICB MHC class I chain-related B molecule

GEO Gene Expression Omnibus

OS Overall survival

IQR Interquartile range

\section{Background}

Colorectal cancer (CRC) is the third most frequently encountered cancer among adults (Siegel et al. 2017; Bray et al. 2018) and is the second in terms of mortality. As a highly heterogeneous disease, tumor immunity plays an important role in CRC.

Immune surveillance can eliminate cancer cells in human body. This process is closely related to major histocompatibility complex and its associated molecules. The human 
major histocompatibility complex (MHC) class I chainrelated genes locate in the HLA class I region of chromosome 6 (Stephens 2001). The MHC class I chain-related B molecule, also commonly known as MICB, is one of the ligands of NKG2D receptor. NKG2D receptors exist in NK cells and $\mathrm{CD} 8^{+} \mathrm{T}$ cells, which mediate antitumor response and immune surveillance (Diefenbach et al. 2001). MICB is expressed by the intestinal epithelium and epithelial tumors as well (Groh et al. 1999).

Cancer cells express MICB as the consequence of cellular stress such as genomic damage (Andrade 2018). MICB can tag these cells for elimination. But tumor cells under antibody-dependent cell-mediated cytotoxicity might develop evasive pathways to avoid NK cell attack (Hu et al. 2019). Shedding is a good way for cancer cells to remove or avoid the surface expression of ligands such as MICB with the presence of metalloproteases in the tumor microenvironment (Schmiedel and Mandelboim 2018). Some previous studies reported that MICB was associated with distant metastasis and advanced stages (Kopp et al. 2009), and associated with rejection to tumors in transplanted mice model (Diefenbach et al. 2001). However, there is still a lack of clinical data confirming the prognostic value of MICB.

Here in this study, we detected MICB expression in CRC tissues, and figured out the relationship between MICB and prognosis in a CRC cohort of Zhongshan Hospital, Fudan University. The prognostic benefit was also validated in a public dataset GSE39582 of GEO database.

\section{Materials and methods}

\section{Study population}

Consecutive CRC patients of Zhongshan Hospital, Fudan University from 2008-05 to 2012-11 were retrospectively enrolled in this study as primary cohort. The inclusion criteria were as follows: receiving primary radical resection, pathologically confirmed colorectal adenocarcinoma, no treatment before surgery, clinicopathological data available. CRC cancer stages were determined according to the International Union Against Cancer (UICC)/American Joint Committee on Cancer (AJCC) TNM classification 8th edition. Radical resections of synchronous liver metastases were also permitted.

The public dataset for validation was selected as: (1) transcriptomic data such as microarray data which include MICB were available; (2) the basic clinical and pathological information including detailed TNM stage and overall survival(OS) was available; (3) the size of dataset was larger than 100; (4) the minimum median follow-up time was 36 months. Thus, GSE39582 dataset was selected from
Gene Expression Omnibus (GEO) repository (Marisa et al. 2013). CRC patients in GSE39582 dataset between 1987 and 2007 were enrolled as validation cohort in the same criteria as primary cohort. And the data of mRNA expression from dataset GSE39582 were also obtained.

This study was approved by the Clinical Research Ethics Committee of Zhongshan Hospital, Fudan University. Informed consent was acquired from all patients of primary cohort for the acquisition of clinical and pathological information and the use of surgical specimens. Since GSE39582 was a public dataset, approval of the ethics committee and informed consent from the patients was unnecessary.

\section{Immunohistochemistry}

For primary cohort, immunohistochemistry was used to detect the MICB expression. The detailed procedure of the experiment was described as previously reported (Mao 2018). The primary antibody was rabbit anti-human polyclonal MICB (diluted 1:100, ARG56879, Arigo). The secondary antibody was goat anti-rabbit. The MICB intensity of +++ was $3,++$ was $2,+$ was 1 , - was 0 . The area score was the percentage of positive cells among all tumor cells multiplied by 100 . Finally, the MICB score was MICB intensity multiplied by area score, ranging from 0 to 300 .

\section{Statistical analysis}

The statistical analyses were performed using the SPSS 25.0. The association between clinicopathological features and MICB were accessed by Chi-square test or Fisher's exact test as appropriate. Kaplan-Meier analysis and Log-rank test were performed to evaluate the relationship between MICB expression and OS. Univariate cox regression analyses were performed to identify the independent prognostic factors among clinicopathological features and other information. Those factors with $P<0.1$ in univariate cox regression analyses were included in the multivariate cox regression analysis. A two-sided $P<0.05$ was considered statistically significant.

The cut-off values of MICB score were calculated for primary and validation cohorts, respectively, because of the different detection method of MICB expression. And the cut-off values were based on the OS data. To obtain the best prognostic efficacy, X-Tile Software (Yale University, version 3.6.1) was used as previously described (Camp et al. 2004). 
Table 1 Baseline clinicopathological characteristics of primary and validation cohorts

\begin{tabular}{|c|c|c|c|c|c|c|}
\hline \multicolumn{3}{|l|}{ Primary cohort } & \multicolumn{3}{|l|}{ Validation cohort } & \multirow[t]{2}{*}{$P$} \\
\hline Factors & No & $\%$ & Factors & No & $\%$ & \\
\hline All patients & 863 & 100.0 & All patients & 556 & 100.0 & \\
\hline Age (years) & & & Age (years) & & & \\
\hline$\leq 60$ & 415 & 48.1 & $\leq 60$ & 157 & 28.2 & $<0.001$ \\
\hline$>60$ & 448 & 51.9 & $>60$ & 398 & 71.6 & \\
\hline Unknown & 0 & 0 & Unknown & 1 & 0.2 & \\
\hline Gender & & & Gender & & & \\
\hline Male & 505 & 58.5 & Male & 306 & 55.0 & 0.196 \\
\hline Female & 358 & 41.5 & Female & 250 & 45.0 & \\
\hline \multicolumn{7}{|l|}{ CEA (ng/ml) } \\
\hline$\leq 5$ & 450 & 52.1 & & & & \\
\hline$>5$ & 413 & 47.9 & & & & \\
\hline Tumor location & & & Tumor location & & & \\
\hline Right-sided colon & 246 & 28.5 & Proximal colon & 218 & 39.2 & $<0.001$ \\
\hline Left-sided colon & 226 & 26.2 & Distal colon & 338 & 60.8 & \\
\hline Rectum & 391 & 45.3 & & & & \\
\hline \multicolumn{7}{|l|}{ Tumor size $(\mathrm{cm})$} \\
\hline$\leq 4.0$ & 478 & 55.4 & & & & \\
\hline$>4.0$ & 385 & 44.6 & & & & \\
\hline \multicolumn{7}{|c|}{ Primary histological type } \\
\hline Non-mucinous & 732 & 84.8 & & & & \\
\hline Mucinous & 131 & 15.2 & & & & \\
\hline \multicolumn{7}{|c|}{ Primary differentiation } \\
\hline Well/moderate & 584 & 67.7 & & & & \\
\hline Poor/anaplastic & 279 & 32.3 & & & & \\
\hline T stage & & & T stage & & & \\
\hline $\mathrm{T} 1$ & 29 & 3.4 & $\mathrm{~T} 1$ & 11 & 2.0 & $<0.001$ \\
\hline $\mathrm{T} 2$ & 120 & 13.9 & $\mathrm{~T} 2$ & 44 & 7.9 & \\
\hline T3 & 207 & 24.0 & T3 & 362 & 65.1 & \\
\hline \multirow[t]{2}{*}{$\mathrm{T} 4$} & 507 & 58.7 & $\mathrm{~T} 4$ & 119 & 21.4 & \\
\hline & & & Unknown & 20 & 3.6 & \\
\hline $\mathrm{N}$ stage & & & $\mathrm{N}$ stage & & & \\
\hline No & 457 & 53.0 & No & 294 & 52.9 & 0.502 \\
\hline N1 & 264 & 30.6 & $\mathrm{~N} 1 / \mathrm{N} 2$ & 242 & 43.5 & \\
\hline $\mathrm{N} 2$ & 142 & 16.4 & Unknown & 20 & 3.6 & \\
\hline \multicolumn{7}{|l|}{ Vascular invasion } \\
\hline No & 761 & 88.2 & & & & \\
\hline Yes & 102 & 11.8 & & & & \\
\hline \multicolumn{7}{|l|}{ Nerve invasion } \\
\hline No & 800 & 92.7 & & & & \\
\hline Yes & 63 & 7.3 & & & & \\
\hline M stage & & & M stage & & & \\
\hline M0 & 642 & 74.4 & M0 & 473 & 85.1 & $<0.001$ \\
\hline \multirow[t]{2}{*}{ M1 } & 221 & 25.6 & M1 & 61 & 11.0 & \\
\hline & & & Unknown & 22 & 4.0 & \\
\hline TNM stage & & & TNM stage & & & \\
\hline I & 114 & 13.2 & I & 32 & 5.8 & $<0.001$ \\
\hline II & 282 & 32.7 & II & 261 & 46.9 & \\
\hline III & 246 & 28.5 & III & 203 & 36.5 & \\
\hline IV & 221 & 25.6 & IV & 60 & 10.8 & \\
\hline
\end{tabular}

Bold values indicate $P<0.05$ 
Table 2 Relationship between MICB and clinical characteristics of primary cohort

\begin{tabular}{|c|c|c|c|}
\hline \multirow[t]{3}{*}{ Factors } & \multicolumn{3}{|c|}{ Primary cohort } \\
\hline & \multicolumn{3}{|c|}{ MICB expression } \\
\hline & Low $(\%)$ & High (\%) & $P$ \\
\hline All patients & 417 & 446 & \\
\hline \multicolumn{4}{|l|}{ Age (years) } \\
\hline$\leq 60$ & $197(47.2)$ & $218(48.9)$ & 0.631 \\
\hline$>60$ & $220(52.8)$ & $228(51.1)$ & \\
\hline \multicolumn{4}{|l|}{ Gender } \\
\hline Male & $240(57.6)$ & $265(59.4)$ & 0.579 \\
\hline Female & $177(42.4)$ & $181(40.6)$ & \\
\hline \multicolumn{4}{|l|}{ CEA (ng/ml) } \\
\hline$\leq 5$ & $222(53.2)$ & $228(51.1)$ & 0.534 \\
\hline$>5$ & $195(46.8)$ & $218(48.9)$ & \\
\hline \multicolumn{4}{|l|}{ Tumor location } \\
\hline Right-sided colon & $119(28.5)$ & $127(28.5)$ & 0.937 \\
\hline Left-sided colon & $107(25.7)$ & $119(26.7)$ & \\
\hline Rectum & $191(45.8)$ & $200(44.8)$ & \\
\hline \multicolumn{4}{|l|}{ Tumor size $(\mathrm{cm})$} \\
\hline$\leq 4.0$ & $227(54.4)$ & $294(65.9)$ & 0.001 \\
\hline$>4.0$ & $190(45.6)$ & $152(34.1)$ & \\
\hline \multicolumn{4}{|c|}{ Primary histological type } \\
\hline Non-mucinous & 329 (78.9) & $403(90.4)$ & $<0.001$ \\
\hline Mucinous & $88(21.1)$ & $43(9.6)$ & \\
\hline \multicolumn{4}{|c|}{ Primary differentiation } \\
\hline Well/moderate & $290(69.5)$ & $294(65.9)$ & 0.255 \\
\hline Poor/anaplastic & $127(30.5)$ & $152(34.1)$ & \\
\hline \multicolumn{4}{|l|}{ T stage } \\
\hline $\mathrm{T} 1 / \mathrm{T} 2$ & $67(16.1)$ & $82(18.4)$ & 0.368 \\
\hline $\mathrm{T} 3 / \mathrm{T} 4$ & $350(83.9)$ & $364(81.6)$ & \\
\hline \multicolumn{4}{|l|}{$\mathrm{N}$ stage } \\
\hline No & $221(53.0)$ & $236(52.9)$ & 0.981 \\
\hline $\mathrm{N} 1 / \mathrm{N} 2$ & $196(47.0)$ & $210(47.1)$ & \\
\hline \multicolumn{4}{|l|}{ Vascular invasion } \\
\hline No & $367(88.0)$ & $394(88.3)$ & 0.880 \\
\hline Yes & $50(12.0)$ & $52(11.7)$ & \\
\hline \multicolumn{4}{|l|}{ Nerve invasion } \\
\hline No & $388(93.0)$ & $412(92.4)$ & 0.706 \\
\hline Yes & $29(7.0)$ & $34(7.6)$ & \\
\hline \multicolumn{4}{|l|}{ M stage } \\
\hline M0 & $302(72.4)$ & $340(76.2)$ & 0.200 \\
\hline M1 & $115(27.6)$ & $106(23.8)$ & \\
\hline \multicolumn{4}{|l|}{ TNM stage } \\
\hline I & $48(11.5)$ & $66(14.8)$ & 0.130 \\
\hline II & $145(34.8)$ & $137(30.7)$ & \\
\hline III & $109(26.1)$ & $137(30.7)$ & \\
\hline IV & $115(27.6)$ & $106(23.8)$ & \\
\hline
\end{tabular}

Bold values indicate $P<0.05$

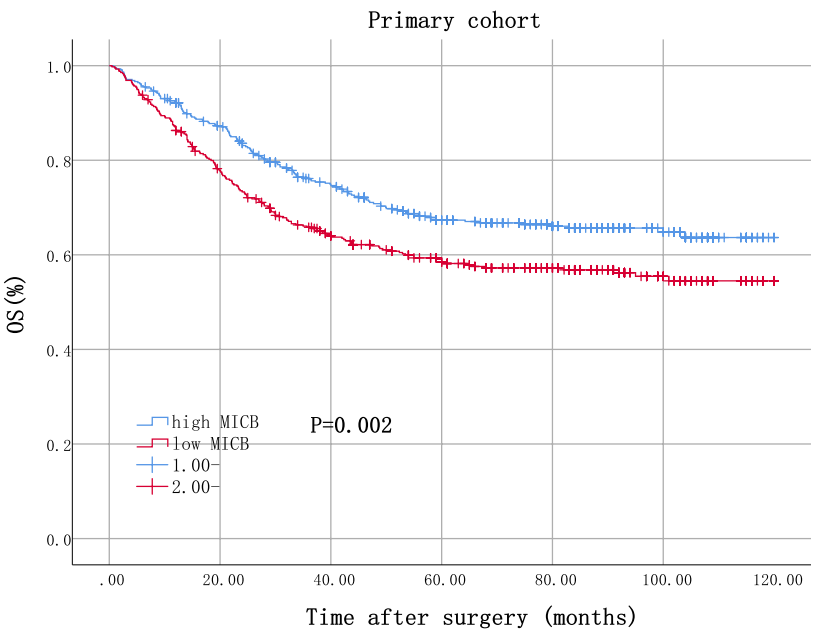

Fig. 1 Kaplan-Meier analysis of primary cohort

\section{Results}

\section{Patient characteristics}

Finally, 863 CRC patients from Zhongshan hospital, Fudan University were enrolled as primary cohort. And 556 patients from dataset GSE39582 were enrolled as validation cohort. The median follow-up time was 60.5 months for primary cohort $(\mathrm{IQR}=24.8-91.0)$ and was 52.0 months (IQR = 27.0-80.0) for validation cohort. 319 (37.0\%) patients of primary cohort died by the time of analysis. And 190 (34.2\%) patients of GSE39582 died by the time of analysis. Baseline clinical and pathological characteristics of primary and validation cohort are presented in Table 1 .

\section{IHC findings}

Representative images of MICB + IHC staining are presented in Supplementary Figure S1. A typical image of $400 \times$ high power field is presented in Supplementary Figure S2.

\section{Definition of cut-off values}

The cut-off value was defined as follows. For MICB expression score detected by IHC in primary cohort, $0-135$ was defined as low, and 136-300 was defined as high. For MICB expression score detected by mRNA in validation cohort, 3.97-6.58 was defined as low, and 6.59-9.53 was defined as high. 
Table 3 Cox regression analyses for OS of primary cohort

\begin{tabular}{|c|c|c|c|c|}
\hline \multirow[t]{3}{*}{ Factors } & \multicolumn{4}{|l|}{ Overall survival } \\
\hline & \multicolumn{2}{|l|}{ Univariate analysis } & \multicolumn{2}{|l|}{ Multivariate analysis } \\
\hline & HR $(95 \%$ CI $)$ & $P$ & HR $(95 \%$ CI $)$ & $P$ \\
\hline \multicolumn{5}{|l|}{ Age (years) } \\
\hline$\leq 60$ & 1 (reference) & 0.644 & & \\
\hline$>60$ & $1.053(0.845-1.312)$ & & & \\
\hline \multicolumn{5}{|l|}{ Gender } \\
\hline Male & 1 (reference) & 0.062 & 1 (reference) & 0.339 \\
\hline Female & $0.805(0.641-1.011)$ & & $0.893(0.708-1.126)$ & \\
\hline \multicolumn{5}{|l|}{ CEA (ng/ml) } \\
\hline$\leq 5$ & 1 (reference) & $<0.001$ & 1 (reference) & 0.003 \\
\hline$>5$ & $2.540(2.017-3.197)$ & & $1.441(1.129-1.840)$ & \\
\hline \multicolumn{5}{|l|}{ Tumor location } \\
\hline Right-sided colon & 1 (reference) & 0.028 & 1 (reference) & 0.183 \\
\hline Left-sided colon & $1.000(0.754-1.326)$ & & $0.854(0.640-1.139)$ & \\
\hline Rectum & $0.737(0.566-0.958)$ & & $0.779(0.596-1.017)$ & \\
\hline \multicolumn{5}{|l|}{ Tumor size $(\mathrm{cm})$} \\
\hline$\leq 4.0$ & 1 (reference) & 0.168 & & \\
\hline$>4.0$ & $1.167(0.937-1.455)$ & & & \\
\hline \multicolumn{5}{|c|}{ Primary histological type } \\
\hline Non-mucinous & 1 (reference) & 0.984 & & \\
\hline Mucinous & $0.997(0.731-1.359)$ & & & \\
\hline \multicolumn{5}{|c|}{ Primary differentiation } \\
\hline Well/moderate & 1 (reference) & $<0.001$ & 1 (reference) & 0.098 \\
\hline Poor/anaplastic & $1.628(1.301-2.038)$ & & $1.213(0.965-1.525)$ & \\
\hline \multicolumn{5}{|l|}{ T stage } \\
\hline $\mathrm{T} 1 / \mathrm{T} 2$ & 1 (reference) & $<0.001$ & 1 (reference) & 0.062 \\
\hline $\mathrm{T} 3 / \mathrm{T} 4$ & $3.198(2.074-4.932)$ & & $1.541(0.978-2.429)$ & \\
\hline \multicolumn{5}{|l|}{$\mathrm{N}$ stage } \\
\hline No & 1 (reference) & $<0.001$ & 1 (reference) & $<0.001$ \\
\hline $\mathrm{N} 1 / \mathrm{N} 2$ & $2.775(2.201-3.499)$ & & $1.752(1.375-2.232)$ & \\
\hline \multicolumn{5}{|l|}{ M stage } \\
\hline M0 & 1 (reference) & $<0.001$ & 1 (reference) & $<0.001$ \\
\hline M1 & $7.922(6.318-9.933)$ & & $6.029(4.718-7.702)$ & \\
\hline \multicolumn{5}{|l|}{ Vascular invasion } \\
\hline No & 1 (reference) & 0.819 & & \\
\hline Yes & $1.041(0.739-1.466)$ & & & \\
\hline \multicolumn{5}{|l|}{ Nerve invasion } \\
\hline No & 1 (reference) & 0.234 & & \\
\hline Yes & $0.755(0.475-1.200)$ & & & \\
\hline \multicolumn{5}{|l|}{ TNM stage } \\
\hline I & 1 (reference) & $<0.001$ & & \\
\hline II & $1.771(0.947-3.315)$ & & & \\
\hline III & $3.005(1.632-5.533)$ & & & \\
\hline IV & $16.535(9.200-29.718)$ & & & \\
\hline \multicolumn{5}{|l|}{ MICB } \\
\hline Low & 1 (reference) & 0.002 & 1 (reference) & 0.008 \\
\hline High & $0.708(0.568-0.883)$ & & $0.741(0.594-0.924)$ & \\
\hline
\end{tabular}

Bold values indicate $P<0.05$ 

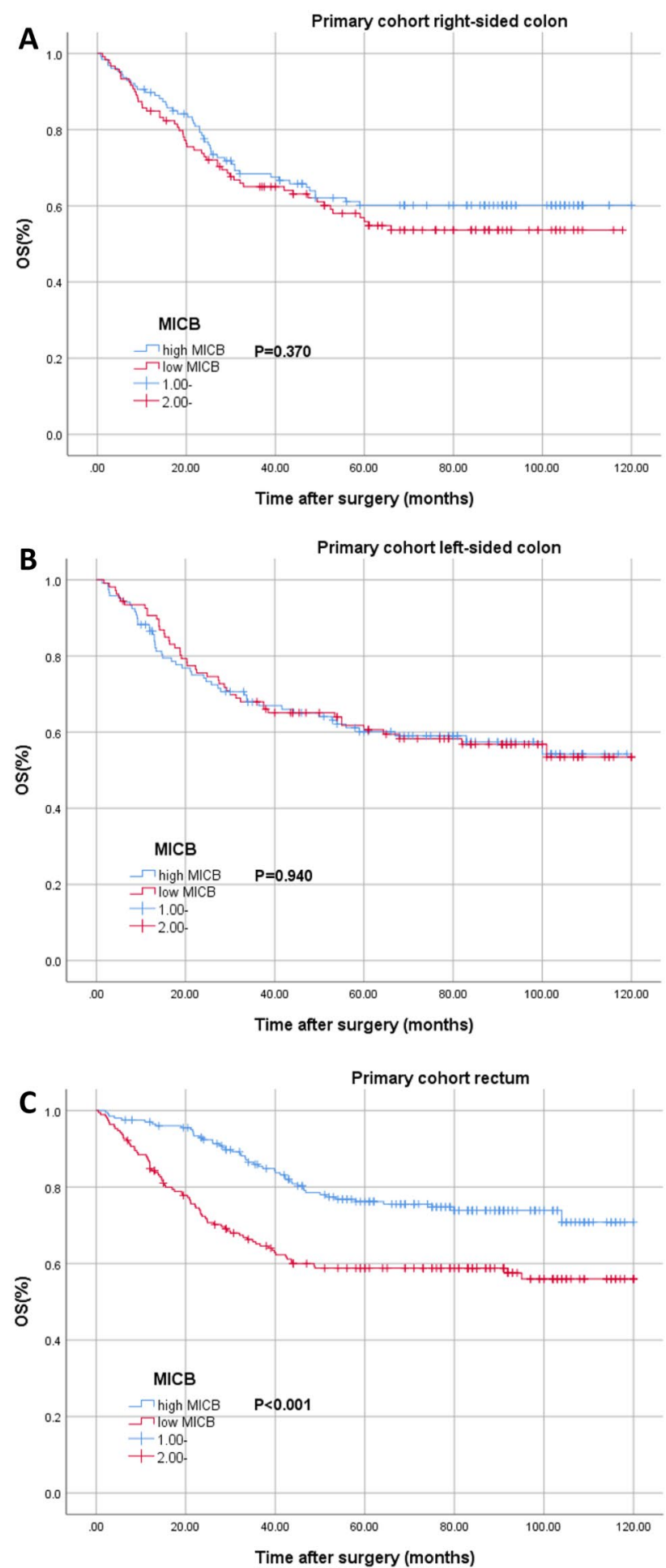

Fig. 2 Kaplan-Meier analysis of patients with right-sided colon (a), left-sided colon (b) and rectal (c) cancer from primary cohort

\section{Association between MICB and clinical characteristics}

The relationship between MICB expression and clinicopathological features of primary cohort is presented in Table 2. High MICB expression was significantly associated with non-mucinous histological type $(P<0.001)$ and tumor size $\leq 4.0 \mathrm{~cm}(P=0.001)$.

The relationship between MICB expression and clinicopathological characteristics of validation cohort is presented in Supplementary Table S1. High MICB expression was significantly associated with tumor located at proximal colon $(P<0.001)$ and M0 stage $(P=0.047)$.

\section{MICB as prognostic biomarkers}

In primary cohort, patients with high MICB expression had significantly better OS ( $P=0.002$, as shown in Fig. 1$)$. In validation cohort, patient with high MICB expression also had significantly better OS $(P=0.001$, as shown in Supplementary Figure S3).

For primary cohort, the univariate cox regression analysis showed that preoperative CEA level, tumor location, primary differentiation, $\mathrm{T}$ stage, $\mathrm{N}$ stage, $\mathrm{M}$ stage and MICB expression were significantly associated with OS. In multivariate analyses, MICB expression, preoperative CEA level, $\mathrm{N}$ stage and $\mathrm{M}$ stage were confirmed as independent prognostic factors for OS (Table 3).

For validation cohort, the univariate analysis showed that age, $\mathrm{T}$ stage, $\mathrm{N}$ stage, $\mathrm{M}$ stage and MICB expression were significantly associated with OS. In multivariate analysis, MICB expression, age and M stage were confirmed as independent prognostic factors for OS (Supplementary Table S2).

\section{Stratified analysis of primary cohort}

In primary cohort, stratified analysis was conducted according to TNM stage. For Stage I and II patients, MICB expression was not a significant prognostic factor $(P=0.214$, Supplementary Figure S4A). For Stage III and IV patients, the survival curves of MICB expression were significant $(P=0.001$, Supplementary Figure S4B). The cox regression showed that MICB expression was a significant independent prognostic factor for OS $(P=0.009$, Supplementary Table S3).

In stratified analysis according to tumor location, for patients with right-sided or left-sided colon cancer, MICB expression was not a significant prognostic factor (Fig. 2a, b). However, for patients with rectal cancer, the survival curves of MICB expression were significant $(P<0.001$, Fig. 2c). And the cox regression showed that MICB expression was a significantly independent prognostic factor for OS $(P<0.001$, Table 4$)$. 
Table 4 Cox regression analyses for OS of patients with rectal cancer from primary cohort

\begin{tabular}{|c|c|c|c|c|}
\hline \multirow[t]{3}{*}{ Factors } & \multicolumn{4}{|l|}{ Overall survival } \\
\hline & \multicolumn{2}{|l|}{ Univariate analysis } & \multicolumn{2}{|l|}{ Multivariate analysis } \\
\hline & HR $(95 \%$ CI $)$ & $P$ & HR $(95 \%$ CI $)$ & $P$ \\
\hline \multicolumn{5}{|l|}{ Age (years) } \\
\hline$\leq 60$ & 1 (reference) & 0.623 & & \\
\hline$>60$ & $1.092(0.769-1.549)$ & & & \\
\hline \multicolumn{5}{|l|}{ Gender } \\
\hline Male & 1 (reference) & 0.453 & & \\
\hline Female & $0.871(0.607-1.250)$ & & & \\
\hline \multicolumn{5}{|l|}{ CEA (ng/ml) } \\
\hline$\leq 5$ & 1 (reference) & $<0.001$ & 1 (reference) & 0.003 \\
\hline$>5$ & $2.346(1.641-3.354)$ & & $1.747(1.209-2.526)$ & \\
\hline \multicolumn{5}{|l|}{ Tumor size (cm) } \\
\hline$\leq 4.0$ & 1 (reference) & 0.306 & & \\
\hline$>4.0$ & $1.203(0.845-1.714)$ & & & \\
\hline \multicolumn{5}{|c|}{ Primary histological type } \\
\hline Non-mucinous & 1 (reference) & 0.701 & & \\
\hline Mucinous & $1.111(0.648-1.906)$ & & & \\
\hline \multicolumn{5}{|c|}{ Primary differentiation } \\
\hline Well/moderate & 1 (reference) & 0.004 & 1 (reference) & 0.098 \\
\hline Poor/anaplastic & $1.667(1.172-2.371)$ & & $1.358(0.945-1.951)$ & \\
\hline \multicolumn{5}{|l|}{ T stage } \\
\hline $\mathrm{T} 1 / \mathrm{T} 2$ & 1 (reference) & $<0.001$ & 1 (reference) & 0.351 \\
\hline $\mathrm{T} 3 / \mathrm{T} 4$ & $2.564(1.538-4.275)$ & & $1.305(0.746-2.282)$ & \\
\hline \multicolumn{5}{|l|}{$\mathrm{N}$ stage } \\
\hline No & 1 (reference) & $<0.001$ & 1 (reference) & 0.013 \\
\hline $\mathrm{N} 1 / \mathrm{N} 2$ & $2.371(1.652-3.404)$ & & $1.606(1.104-2.336)$ & \\
\hline \multicolumn{5}{|l|}{ M stage } \\
\hline M0 & 1 (reference) & $<0.001$ & 1 (reference) & $<0.001$ \\
\hline M1 & $5.494(3.856-7.828)$ & & $4.303(2.976-6.223)$ & \\
\hline \multicolumn{5}{|l|}{ Vascular invasion } \\
\hline No & 1 (reference) & 0.360 & & \\
\hline Yes & $1.269(0.761-2.116)$ & & & \\
\hline \multicolumn{5}{|l|}{ Nerve invasion } \\
\hline No & 1 (reference) & 0.840 & & \\
\hline Yes & $0.935(0.490-1.785)$ & & & \\
\hline \multicolumn{5}{|l|}{ TNM stage } \\
\hline I & 1 (reference) & $<0.001$ & & \\
\hline II & $1.595(0.755-3.368)$ & & & \\
\hline III & $2.559(1.272-5.146)$ & & & \\
\hline IV & $9.924(5.058-19.473)$ & & & \\
\hline \multicolumn{5}{|l|}{ MICB } \\
\hline Low & 1 (reference) & $<0.001$ & 1 (reference) & $<0.001$ \\
\hline High & $0.492(0.343-0.705)$ & & $0.510(0.356-0.733)$ & \\
\hline
\end{tabular}

Bold values indicate $P<0.05$

\section{Discussion}

In this study, a large cohort of real world was constructed as primary cohort to evaluate the prognostic benefit of MICB in colorectal cancer patients. The positive association between MICB and OS was found in our cohort. And MICB was also identified as a new independent prognostic indicator of $\mathrm{OS}$ in CRC patients. Then, the prognostic value of MICB was also validated in GES39582, which is a public GEO cohort. 
Stratified analysis indicated that our results are more useful for patients with rectal cancer. This may be related to the rupture of rectal wall caused by feces. As a result, more tumor antigens enter the systemic circulation and stimulate the immune response. Stratified analysis also suggested that stage III and IV patients with high MICB expression had a better prognosis. This may be related to lymph node metastasis, further stimulating the immune response.

The potential mechanism of MICB as a prognostic indicator in CRC patients might be explained by a previous study. Overexpression of microRNA, including miR-17-5p, miR-20a, miR-93, miR-106b, miR-372, miR-373 and miR$520 \mathrm{c}$, resulted in downregulation of MICB expression on the surface of cancer cells and less susceptibility to NKG2Ddependent killing by NK cells (Stern-Ginossar et al. 2008). Eventually, the downregulation of MICB expression enables the tumor to avoid immune recognition which explains the reason of a worse OS in low MICB patients with CRC. Asking for the therapeutic value of MICB, Ferrari de Andrade et al. found that MICB a3 domain-specific antibodies substantially increased the density of the stimulatory MICB ligands on the surface of cancer cells, reduced shed MICB amounts, and induced NK cell attack against cancer cells (Andrade 2018). This suggests that elevating MICB level could be a potential therapy for CRC patients.

However, a couple of limitations of this study must be noticed. First, our study is a retrospective one. To further validate our conclusion, a prospective study with data from multiple centers is necessary, especially for patients with rectal cancer as well as stage III and IV patients. Second, MICB intensity and area score were not detected and determined automatically, resulting in potential artificial errors. Third, the MICB detection method of GSE39582 is different from the method of primary cohort. And the cut-off value of MICB expression was not fully verified.

\section{Conclusion}

In summary, MICB was identified as a new independent prognostic factor for CRC patients. CRC with high MICB expression conferred survival benefit. This could promote the individualized treatment of colorectal cancer.

Author contributions QF designed the work. SY analyzed and interpreted the patient data. YM performed the immunohistochemistry examinations. QF, SY, YM and MJ were major contributors in writing the manuscript. QF, SY, YM and MJ contributed equally to this article as the co-first authors. All authors read and approved the final manuscript.
Funding National Natural Science Foundation of China (81602040), Excellent Youth Talent Development Program of Zhongshan Hospital, Fudan University (2019ZSYXQN03).

Data availability The datasets used and analyzed during the current study are available from the corresponding author on reasonable request.

\section{Compliance with ethical standards}

Conflict of interest The authors declare that they have no competing interests.

Ethical approval This study was approved by the Clinical Research Ethics Committee of Zhongshan Hospital, Fudan University.

Informed consent Informed consent was acquired from all patients of primary cohort for the acquisition of clinical and pathological information and the use of surgical specimens.

Open Access This article is licensed under a Creative Commons Attribution 4.0 International License, which permits use, sharing, adaptation, distribution and reproduction in any medium or format, as long as you give appropriate credit to the original author(s) and the source, provide a link to the Creative Commons licence, and indicate if changes were made. The images or other third party material in this article are included in the article's Creative Commons licence, unless indicated otherwise in a credit line to the material. If material is not included in the article's Creative Commons licence and your intended use is not permitted by statutory regulation or exceeds the permitted use, you will need to obtain permission directly from the copyright holder. To view a copy of this licence, visit http://creativecommons.org/licenses/by/4.0/.

\section{References}

Bray F, Ferlay J, Soerjomataram I et al (2018) Global cancer statistics 2018: GLOBOCAN estimates of incidence and mortality worldwide for 36 cancers in 185 countries. CA Cancer J Clin 68(6):394-424

Camp RL, Dolled-Filhart M, Rimm DL (2004) X-tile: a new bioinformatics tool for biomarker assessment and outcome-based cut-point optimization. Clin Cancer Res 10:7252-7259

de Andrade F et al (2018) Antibody-mediated inhibition of MICA and MICB shedding promotes NK cell-driven tumor immunity. Science 359:1537-1542

Diefenbach A, Jensen ER, Jamieson AM, Raulet DH (2001) Rae1 and H60 ligands of the NKG2D receptor stimulate tumor immunity. Nature 413(6852): 165-171

Groh V, Rhinehart R, Secrist H, Bauer S, Garbstein KH, Spies T (1999) Broad tumor-associated expression and recognition by tumorderived $\gamma \delta$ T cells of MICA and MICB. Proc Natl Acad Sci USA 96:6879-6884

Hu X, Zhang J, Wang J et al (2019) Landscape of B cell immunity and related immune evasion in human cancers. Nat Genet. https://doi. org/10.1038/s41588-018-0339-x

Kopp R, Glas J, Lau-Werner U, Albert ED, Weiss EH (2009) Association of MICA-TM and MICB C1_2_A microsatellite polymorphisms with tumor progression in patients with colorectal cancer. J Clin Immunol 29:545-554

Mao Y et al (2018) Low tumor infiltrating mast cell density confers prognostic benefit and reflects immunoactivation in colorectal cancer. Int J Cancer 143(9):2271-2280 
Marisa L, de Reynies A, Duval A et al (2013) Gene expression classification of colon cancer into molecular subtypes: characterization, validation, and prognostic value. PLoS Med 10:e1001453

Schmiedel D, Mandelboim O (2018) NKG2D ligands-critical targets for cancer immune escape and therapy. Front Immunol 9:2040. https://doi.org/10.3389/fimmu.2018.02040

Siegel RL, Miller KD, Fedewa SA et al (2017) Colorectal cancer statistics, 2017. CA Cancer J Clin 67:177-193

Stephens HA (2001) MICA and MICB genes: can the enigma of their polymorphism be resolved. Trends Immunol 22(7):378-385
Stern-Ginossar N, Gur C, Biton M et al (2008) Human microRNAs regulate stress-induced immune responses mediated by the receptor NKG2D. Immunol Nat. https://doi.org/10.1038/ni.1642

Publisher's Note Springer Nature remains neutral with regard to jurisdictional claims in published maps and institutional affiliations. 